Bio - grafía. Escritos sobre la Biología y su Enseñanza. ISSN 2027-1034

Edición Extraordinaria. p.p. 1078 - 1087

Memorias del IX Encuentro Nacional de Experiencias en Enseñanza de la Biología y la Educación Ambiental. IV Congreso Nacional de Investigación en Enseñanza de la Biología.

\title{
TRAT AMIENTO DIDÁCTICO DEL CONCEPTO BIODIVERSIDAD EN ESPACIOS NO FORMALES DE EDUCACIÓN: UNA MIRADA DESDE LA INVESTIGACIÓN EN EL CAMPO
}

\section{DIDACTIC TREATMENT OF THE CONCEPT OF BIODIVERSITY IN NON-FORMAL EDUCATION SPACES: A FIELDWORK-BASED PERSPECTIVE}

\author{
Yonier Alexander Orozco Marín ${ }^{1}$
}

\section{RESUMEN}

La enseñanza de la biodiversidad ha venido constituyéndose como prioridad en diferentes escenarios educativos formales y no formales. Actualmente se reconoce la necesidad de formar ciudadanos sean conscientes de la importancia de la biodiversidad y de posibles estrategias individuales y colectivas para su conservación. Los museos, zoológicos, acuarios, entre otros espacios vienen implementando estrategias para divulgar conocimientos sobre la biodiversidad a variados públicos, generando un campo de investigación que se pregunta por los procesos de enseñanza y aprendizaje de este concepto en estos espacios. El objetivo del presente texto fue el de caracterizar el abordaje didáctico dado al concepto de biodiversidad en espacios de educación no formal, a través del análisis de investigaciones que tuvieron como objeto de estudio, de manera explícita, el abordaje del concepto en el sistema didáctico interno y/o externo de diferentes espacios de educación no formal. Fueron analizadas 18 investigaciones, identificando los siguientes elementos de análisis: La transposición museográfica del concepto y la adecuación del espacio pensando en la experiencia del visitante, en el sistema didáctico interno. En el sistema didáctico externo fueron identificados la búsqueda de evidencias de aprendizaje en diversos públicos, el proceso de aprendizaje en la visita, el papel del mediador y la relación escuela- espacio no formal de educación. Se defiende la necesidad de profundizar en este campo de investigación con el fin de fortalecer la dimensión educativa de estos espacios.

PALABRAS CLAVE: Concepto biodiversidad, Educación en Museos y Zoológicos, Educación no formal, Enseñanza de la biología, Espacios de educación no formal.

\footnotetext{
1 Discente Mestrado Profissional em Ensino de Ciências e Matemáticas, Universidade Federal do Acre, Rio Branco (A-Brasil). apmusicomano@gmail.com
} 
Bio - grafía. Escritos sobre la Biología y su Enseñanza. ISSN 2027-1034

Edición Extraordinaria. p.p. 1078 - 1087

Memorias del IX Encuentro Nacional de Experiencias en Enseñanza de la Biología y la

Educación Ambiental. IV Congreso Nacional de Investigación en Enseñanza de la Biología.

\begin{abstract}
Teaching biodiversity has been a priority in different formal and non-formal education scenarios. It is now recognized that citizens are aware of the importance of biodiversity and possible individual and collective strategies for their conservation. Museums, zoos, aquariums, among other spaces have been implementing strategies to disseminate knowledge about biodiversity to various publics, generating a field of research that asks about the teaching and learning processes of this concept in these spaces. The objective of the present work was to characterize the didactic approach given to the concept of biodiversity in non-formal education spaces, through the analysis of research that had as object of study, explicitly, the approach of the concept in the internal didactic system And / or outside of different non-formal education spaces. 18 researches were analyzed, identifying the following elements of analysis: The museographic transposition of the concept and the adequacy of the space, considering the experience of the visitor, in the internal didactic system. In the external didactic system were identified: The search for evidence of learning in different audiences, the learning process in the visit, the role of the mediator and the non-formal school-space relationship of education. It is defended the need to deepen this field of research in order to strengthen its educational dimension.
\end{abstract}

KEY-WORDS: Biodiversity concept, Biology education, Education in Museums and Zoos, Non-formal education, Non-formal education spaces.

\title{
INTRODUCCIÓN
}

Actualmente es reconocida la función educativa de los espacios no formales de educación, tales como, museos, zoológicos, acuarios, centros de ciencias, entre otros. Esa dimensión educativa no consiste únicamente en la divulgación de contenidos de las ciencias a los visitantes, comprende significados más amplios donde se construyen aprendizajes que no siempre son de carácter cognitivo, más relacionados hacia la promoción de la interacción social, incentivar el cuestionamiento o estimular la curiosidad. De Genzini y Almeida (2013) apuntan que esos aspectos pueden contribuir en la adquisición de competencias para el aprendizaje de conceptos científicos.

En los espacios formales y no formales de educación los conocimientos científicos no son presentados de la misma manera que son producidos por las comunidades científicas, pasan por procesos denominados como transposición o recontextualización. Marandino (2004) propone el término de transposición museográfica para denominar al conjunto de diversas transformaciones de conocimientos que pueden acontecer en el sistema didáctico museal interno y el sistema didáctico museal externo (Figura 1). 
Bio - grafía. Escritos sobre la Biología y su Enseñanza. ISSN 2027-1034

Edición Extraordinaria. p.p. 1078 - 1087

Memorias del IX Encuentro Nacional de Experiencias en Enseñanza de la Biología y la Educación Ambiental. IV Congreso Nacional de Investigación en Enseñanza de la Biología.

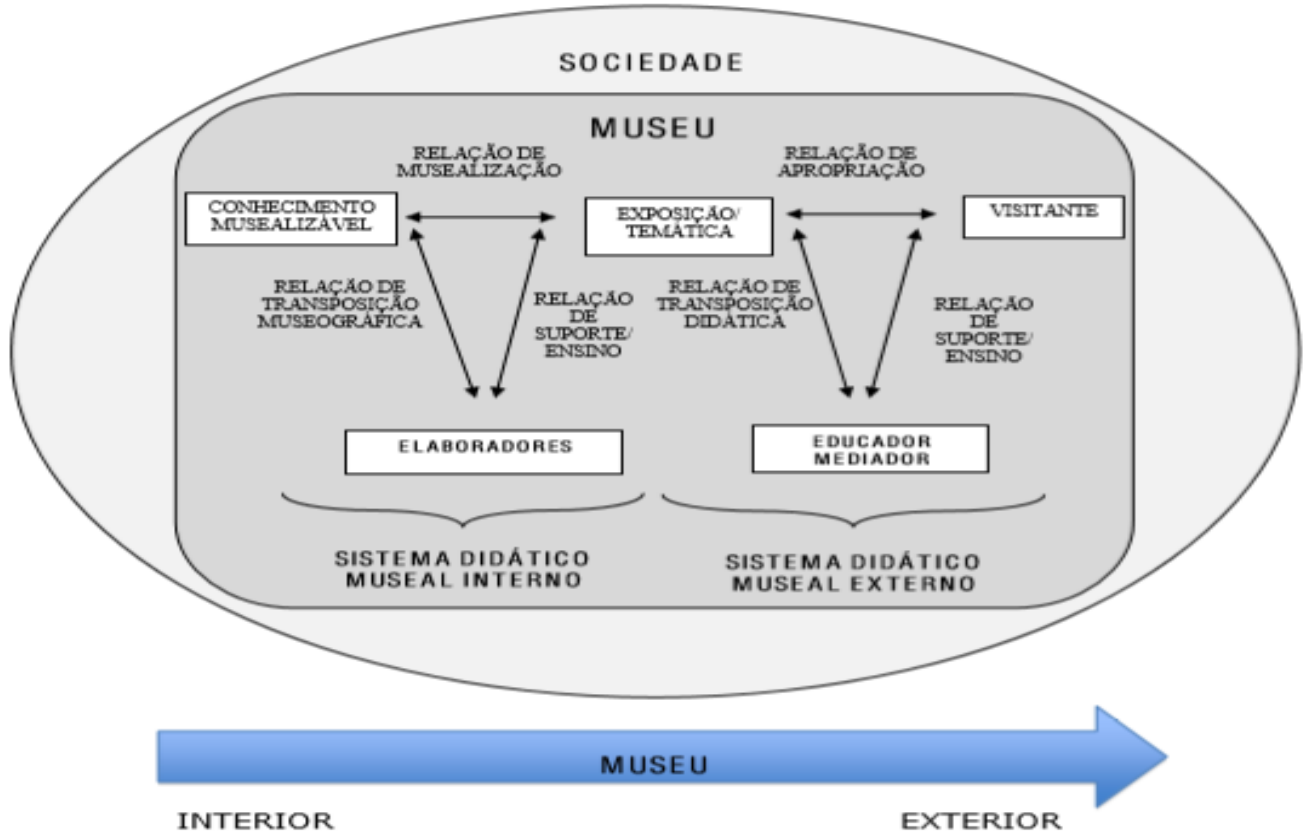

Figura 1. Transposición Museográfica en el sistema didáctico museal interno y sistema didáctico museal externo. Marandino (2011) "Por uma didática museal; Propondo bases sociológicas e epistemológicas para a análise da educação em museus".

En el sistema didáctico museal interno acontece el proceso de construcción de la exposición temática, teniendo como base el "conocimiento musealizable" y donde los elaboradores tienen un papel fundamental en su interpretación. En el sistema didáctico museal externo se desarrolla concretamente el proceso de enseñanza y aprendizaje a través de la presentación de la exposición temática y de la propuesta de actividades a los visitantes, experiencias en algunos casos orientadas por la figura del mediador. Para esta investigación, fue considerado el sistema didáctico museal interno y externo como marco de referencia para analizar el abordaje didáctico del concepto biodiversidad en diversos espacios de educación no formal.

La biodiversidad es un concepto relativamente reciente, propuesto por Edward Wilson (1988) y que a lo largo de estas tres décadas ha transcendido los límites de la biología y la ecología para ser discutido y abordado desde perspectivas políticas, culturales, sociales y económicas. Biológica y ecológicamente se ha definido como un concepto que se refiere a la variedad de la vida producto de la evolución, una propiedad de la vida que puede ser identificada en tres niveles jerárquicos de organización: nivel genético, nivel de las especies y nivel ecosistémico. Desde otras perspectivas de explicación, se menciona la interdependencia existente entre la diversidad biológica y la diversidad cultural, el valor 
Bio - grafía. Escritos sobre la Biología y su Enseñanza. ISSN 2027-1034

Edición Extraordinaria. p.p. 1078 - 1087

Memorias del IX Encuentro Nacional de Experiencias en Enseñanza de la Biología y la

Educación Ambiental. IV Congreso Nacional de Investigación en Enseñanza de la Biología.

económico de la biodiversidad, las tensiones y diálogos sobre su gestión en escenarios políticos y la necesidad de valorizar el patrimonio inmaterial de diferentes culturas (sus conocimientos y tradiciones) como alternativa para la conservación.

Sobre el abordaje del concepto biodiversidad en espacios no formales de educación, una investigación realizada con 5561 visitantes de 26 zoológicos distribuidos en 19 países (Moss, Jensen \& Gusset, 2014) reportó que después de visitar un zoológico, los visitantes incrementan su entendimiento sobre lo que es biodiversidad, así como sus conocimientos sobre acciones para contribuir en su protección. Estos datos justifican la importancia de estudiar el abordaje didáctico dado al concepto en estos espacios. El objetivo del presente trabajo fue el de caracterizar el abordaje didáctico dado al concepto de biodiversidad en museos, zoológicos, acuarios y otros espacios de educación no formal, a través del análisis de investigaciones que tuvieron como objeto de estudio y de manera explícita, el abordaje del concepto en el sistema didáctico interno y/o externo de diferentes espacios de educación no formal.

\section{METODOLOGÍA}

La investigación se orientó desde el enfoque metodológico de la metodología de análisis de contenido, desde una perspectiva cualitativa (Moraes, 1999). Inicialmente se realizó la búsqueda de los trabajos, haciendo uso de los siguientes términos de búsqueda: Biodiversidad Zoológicos, Biodiversidad Museos, Biodiversidad acuarios, Biodiversidad espacios de educación no formal. Los cuatro términos fueron traducidos al portugués y al inglés, a fin de realizar la búsqueda en el programa Google scholar. La búsqueda fue complementada en las memorias de los Encuentros Nacionales de Pesquisa en educación en Ciencias (ENPECs) y los Encuentros Nacionales de Enseñanza de la biología (ENEBIOs), ambos encuentros realizados en Brasil, país donde la educación en espacios no formales de aprendizaje viene fortaleciéndose como línea de investigación y producción de conocimiento.

Fueron analizados los trabajos que abordaban explícitamente el concepto biodiversidad desde la dimensión educativa de esos espacios. No fueron analizados trabajos que estudiaban la biodiversidad en espacios de educación no formal en otras dimensiones diferentes a la dimensión educativa de la Institución, tampoco los trabajos que trataban conceptos familiares como medio ambiente, naturaleza, entre otros. Fueron seleccionados 18 trabajos para análisis, producidos entre el 2005 y el 2016. Siendo la gran mayoría realizados en museos, seguidos de los trabajos realizados en Zoológicos y acuarios. Investigaciones realizadas en Centros de ciencias (Biodomo), Jardines Botánicos, Parques Ecológicos y espacios de educación no formal no institucionalizados también fueron encontradas, correspondiendo un trabajo a cada espacio. Gran parte de los trabajos fueron realizados en Instituciones de Brasil, seguidas de Colombia, Canadá e Inglaterra. Investigaciones de Austria, Hungría, Israel y Malta también fueron analizadas. 
Bio - grafía. Escritos sobre la Biología y su Enseñanza. ISSN 2027-1034

Edición Extraordinaria. p.p. 1078 - 1087

Memorias del IX Encuentro Nacional de Experiencias en Enseñanza de la Biología y la Educación Ambiental. IV Congreso Nacional de Investigación en Enseñanza de la Biología.

Fue posible organizar los trabajos en dos grupos, el primero correspondiente a los trabajos que tenían por objeto de estudio el tratamiento de la biodiversidad en el sistema didáctico interno, y en el segundo, los que estudiaron el abordaje del concepto en el sistema didáctico externo. Se realizó lectura detallada de cada conjunto de trabajos a fin de identificar aspectos preliminares de análisis, dando como resultado un número extenso de aspectos identificados. Establecidos estos aspectos preliminares se procedió a establecer agrupaciones finales más amplias y genéricas.

\section{RESULTADOS Y DISCUSIÓN}

Tabla 1. Número de trabajos y objetos de estudio identificados sobre el abordaje didáctico de la biodiversidad en espacios de educación no formal.

\begin{tabular}{|c|c|c|}
\hline $\begin{array}{l}\text { Aspectos de } \\
\text { análisis }\end{array}$ & $\begin{array}{l}\text { Sistema didáctico museal } \\
\text { interno }\end{array}$ & Sistema didáctico museal externo \\
\hline $\begin{array}{l}\text { Número de } \\
\text { trabajos }\end{array}$ & 7 & 11 \\
\hline $\begin{array}{l}\text { Objetos de } \\
\text { estudio } \\
\text { identificados }\end{array}$ & $\begin{array}{l}\text {-La transposición } \\
\text { museográfica del concepto } \\
\text { biodiversidad. } \\
\text {-La adecuación del espacio } \\
\text { de educación no formal } \\
\text { pensando en la experiencia } \\
\text { de los visitantes }\end{array}$ & $\begin{array}{c}\text {-Las evidencias de aprendizajes sobre } \\
\text { biodiversidad en diversos públicos. } \\
\text {-El proceso de aprendizaje durante } \\
\text { una visita. } \\
\text {-El papel del mediador. } \\
\text {-El vínculo entre la educación formal y } \\
\text { no formal }\end{array}$ \\
\hline
\end{tabular}

Sistema didáctico interno: ¿Cómo se presenta la biodiversidad en las exposiciones temáticas de los espacios no formales de educación?

De los dieciocho trabajos analizados, siete tuvieron como foco de estudio el abordaje del concepto biodiversidad en el sistema didáctico interno (Tabla 1).

\section{Transposición "Museográfica" del concepto}

Los resultados de las investigaciones analizadas muestran que el concepto de biodiversidad que se encuentra en manuales didácticos de ecología usados en universidades, aborda el concepto a través de la explicación de sus tres niveles de organización, desde perspectivas biogeográficas y evolutivas principalmente, abordando también el impacto del hombre sobre la biodiversidad. A través del proceso de transposición "museográfica", en los espacios no formales de educación, la biodiversidad 
Bio - grafía. Escritos sobre la Biología y su Enseñanza. ISSN 2027-1034

Edición Extraordinaria. p.p. 1078 - 1087

Memorias del IX Encuentro Nacional de Experiencias en Enseñanza de la Biología y la Educación Ambiental. IV Congreso Nacional de Investigación en Enseñanza de la Biología.

es presentada principalmente desde una perspectiva conservacionista de las especies y sus hábitats (De Mattos, 2011). Dentro del ámbito de explicación biológico-ecológico, el nivel genético de la biodiversidad es abordado en menor medida que el nivel de organización de las especies y de los ecosistemas (Marandino \& Diaz, 2011).

Si bien, los espacios de educación no formal empiezan a profundizar en esfuerzos por abordar la biodiversidad desde perspectivas culturales, políticas y socio-económicas, este aspecto aún representa desafíos. Abordar estos aspectos es importante, considerando que la puesta en marcha de acciones concretas para la conservación de la biodiversidad en determinado contexto, depende de la disponibilidad de conocimientos sobre la biodiversidad de la región, de las prioridades políticas para la conservación, la disponibilidad de recursos económicos y las prácticas culturales y educativas asociadas a esa biodiversidad. El estudio de las concepciones de los elaboradores de las exposiciones y la discusión sobre su formación vienen ganando importancia también, sugiriendo la necesidad de la formación de equipos interdisciplinares para la elaboración de exposiciones temáticas, fundamentadas no únicamente desde la perspectiva de los expertos, también de los visitantes.

\section{Promoviendo interacciones: Adecuación del Espacio pensando en la experiencia del visitante}

Los espacios no formales de educación superan la preocupación de reproducir o divulgar fielmente conceptos producidos por la comunidad científica. Si bien, son abordadas diversas temáticas con diversos públicos, la promoción del entretenimiento y la interacción social, la divulgación del patrimonio natural-cultural y la estimulación de la curiosidad y el cuestionamiento también son prioridad en estos espacios.

Atender estos objetivos condiciona a estas instituciones a planear exposiciones temáticas llamativas para los visitantes, en las que estos se sumerjan en experiencias envolventes y estimulantes de la reflexión y la participación. Para el caso particular de experiencias asociadas al concepto biodiversidad, las investigaciones identifican "objetos destacados" en algunas exposiciones que llaman la atención de los visitantes antes y durante la visita (Kistler \& Marandino, 2015). Algunos museos construyen dioramas, los cuales son representaciones a escala de las especies en contextos más reales. Los zoológicos y acuarios, por su parte, adelantan esfuerzos por dar mejores condiciones de bienestar a su colección a través de la representación de ecosistemas con sus características originales. Se pretende que la presencia de estos objetos e instalaciones estimulen a los visitantes a interactuar desde diferentes sentidos, interactuar más con el mediador y/o con sus compañeros de visita.

\section{Sistema didáctico externo: Experiencias de enseñanza y de aprendizaje sobre biodiversidad.}

Once trabajos tuvieron su foco de estudio en el sistema didáctico externo (Tabla 1), donde la exposición temática es presentada al visitante. 
Bio - grafía. Escritos sobre la Biología y su Enseñanza. ISSN 2027-1034

Edición Extraordinaria. p.p. 1078 - 1087

Memorias del IX Encuentro Nacional de Experiencias en Enseñanza de la Biología y la Educación Ambiental. IV Congreso Nacional de Investigación en Enseñanza de la Biología.

\section{Buscando evidencias de contribución de los espacios de educación no formal a los aprendizajes de diversos públicos}

Todas las investigaciones que trataron este aspecto coinciden en que a través de las visitas a estas Instituciones, diversos públicos "aumentaron" o desarrollaron lo que de manera general podríamos llamar de aprendizajes sobre la biodiversidad. Sin embargo, fue posible identificar la amplia heterogeneidad de denominaciones atribuidas en las investigaciones a los "resultados de aprendizaje" de los visitantes. Entendimientos, resignificaciones, reflexiones, aprendizajes, actitudes y comprensiones fueron algunos de los términos utilizados para referirse a las categorías desde las cuales fueron estudiados, explícitamente o no, esos resultados.

Fue posible identificar dos perspectivas epistemológicas que fundamentaron las caracterizaciones de los "resultados de aprendizaje" sobre la biodiversidad de los visitantes. En algunos trabajos el aprendizaje es concebido como un resultado cuantificable, donde generalmente se compara la cantidad de información que el visitante "conocía" antes de la visita sobre biodiversidad y la que "conoce" después (Moss, Jensen \& Gusset, 2014). Por otra parte, algunos trabajos conciben el aprendizaje como un proceso socio-interactivo, donde son caracterizadas las concepciones sobre biodiversidad construidas por los visitantes o las actitudes a corto y mediano plazo que fueron objeto de reflexión (López, 2011).

Algunos investigaciones encontraron que las reflexiones manifestadas por los visitantes sobre la biodiversidad no siempre se relacionan directamente con las actividades propuestas, ni su contenido temático (Angulo, 2011). Los trabajos sugieren la necesidad de implementar esfuerzos por conocer las expectativas de los visitantes, a fin de construir exposiciones temáticas con objetivos de aprendizaje más adaptados a las características del público, caso de un zoológico en Israel que construyó una exposición sobre la conservación de la biodiversidad asociada a innovaciones tecnológicas, a partir de la caracterización de los conocimientos sobre biodiversidad de diversos visitantes (Topaz, 2016).

\section{Foco en el proceso: ¿Cómo actúan y aprenden los visitantes en estos espacios?}

Estas investigaciones ubican su foco en el "proceso de aprendizaje" en los espacios de educación no formal. Considerando el aprendizaje desde perspectivas socio-interactivas, los resultados de estos resaltan la importancia de promover conversaciones e intercambio de ideas sobre la conservación de la biodiversidad. Las conversaciones de los visitantes son generalmente afectivas y emotivas (De Queiroz \& Fernandes, 2015), de ahí la necesidad de proponer experiencias que estimulen también conversaciones conectivas entre el contenido temático de la exposición y la experiencia cotidiana del visitante.

También ha sido identificado que los objetos de estos espacios solo ganan verdadero significado a través del tipo de interacción que el sujeto establece con él. Así mismo, se 
Bio - grafía. Escritos sobre la Biología y su Enseñanza. ISSN 2027-1034

Edición Extraordinaria. p.p. 1078 - 1087

Memorias del IX Encuentro Nacional de Experiencias en Enseñanza de la Biología y la

Educación Ambiental. IV Congreso Nacional de Investigación en Enseñanza de la Biología.

ha encontrado que el tipo de actividad propuesta tiene una relación directa con el tipo de aprendizaje desarrollado por el visitante: motivacional, creativo o conceptual (Kimble, 2013).

\section{ANALIZANDO EL PAPEL DEL MEDIADOR EN LA EXPERIENCIA DE ENSEÑANZA Y APRENDIZAJE.}

Si bien el papel del mediador ha sido ampliamente discutido principalmente en la investigación en educación en museos, ha sido un aspecto abordado en menor medida en otros espacios no formales de educación. Los trabajos destacan la necesidad de problematizar la formación inicial y continuada del mediador (De Oliveira \& Franco, 2014). Cuando su formación privilegia aspectos disciplinares (biología, ecología, zoología) sobre otros aspectos necesarios para entender y desarrollar procesos de enseñanza de la biodiversidad (didáctica, pedagogía), el lenguaje del cual el mediador acaba haciendo uso frecuentemente es altamente técnico, sin una adaptación a las características y necesidades de los visitantes. La formación de equipos conformados por mediadores formados en diferentes áreas del conocimiento aparece como una tendencia en algunos espacios de educación no formal, con el fin de abordar la conservación de la biodiversidad desde perspectivas más holísticas.

\section{El vínculo entre la educación formal y no formal.}

El uso de espacios de educación no formal por parte de docentes para abordar el concepto de biodiversidad con grupos escolares es considerado importante y relevante desde la educación formal. Con todo, parece importante profundizar en la investigación sobre la relación entre espacios formales y no formales de educación para la enseñanza de la biodiversidad y su conservación al público escolar. Se ha identificado el papel destacado que juegan las ideas previas de los alumnos en estos espacios, la necesidad de una adecuada planeación previa a la visita y una reflexión en el aula posterior a ella, así como la necesidad de abordar la educación en espacios no formales de educación desde la formación inicial del docente de biología y ciencias naturales.

\section{CONCLUSIONES}

Es posible destacar la necesidad de seguir profundizando en la investigación de los procesos de enseñanza y aprendizaje de la biodiversidad y su conservación en espacios formales y no formales de educación. Principalmente en el contexto latinoamericano, caracterizado por la variedad de ecosistemas, la diversidad de especies, recursos genéticos y diversidad de prácticas culturales asociados a esa biodiversidad. Este trabajo contribuyó a definir ejes temáticos que pueden posibilitar el desarrollo sistemático de la investigación en este campo.

Los espacios de educación no formal son Instituciones que ofrecen la oportunidad de participación de toda la ciudadanía en el reconocimiento de su biodiversidad y la reflexión sobre estrategias de su conservación, por lo tanto, se hace necesario desarrollar 
Bio - grafía. Escritos sobre la Biología y su Enseñanza. ISSN 2027-1034

Edición Extraordinaria. p.p. 1078 - 1087

Memorias del IX Encuentro Nacional de Experiencias en Enseñanza de la Biología y la Educación Ambiental. IV Congreso Nacional de Investigación en Enseñanza de la Biología.

investigaciones que permitan fortalecer su dimensión educativa y la innovación en estrategias didácticas más contextualizadas a las características de la biodiversidad del contexto regional (Orozco, 2016) y de sus visitantes.

En los espacios de educación no formal, la presentación de la biodiversidad se relaciona principalmente con la conservación de especies y ecosistemas, incluyen aspectos biológicos-ecológicos en interacción con elementos culturales de la biodiversidad. Se encuentra el desafío de construir propuestas y objetos que estimulen al visitante a problematizar el medio y no solo contemplarlo, es decir, vincular al visitante en experiencias más activas orientadas a la reflexión y construcción de nuevas ideas. Las investigaciones analizadas respaldan la idea de que los espacios de educación no formal favorecen el aprendizaje sobre la biodiversidad y estrategias para su conservación en diversos públicos, sin embargo, la concepción de aprendizaje presente en las investigaciones es bastante heterogénea. Una línea interesante de investigación privilegia la caracterización del proceso de aprendizaje del visitante más que los resultados de aprendizaje sobre la biodiversidad, estas investigaciones permiten obtener resultados importantes para reorientar las actividades de estas Instituciones. La relación entre la educación formal y no formal, así como la formación de los mediadores de estos espacios para la enseñanza de la biodiversidad son líneas de estudio representadas por pocas investigaciones.

\section{REFERENCIAS}

Angulo, F. (2011). Hacia el desarrollo de una sociedad alfabetizada ambientalmente. El caso del Museo Interactivo de EPM. Informe técnico de investigación. Universidad de Antioquia.

De Mattos, M. (2011). A transposição Museográfica da Biodiversidade no Aquário de Ubatuba: Estudo através de mapas conceituais. Dissertação (Mestrado em educação) - Universidade de São Paulo. São Paulo.

De Oliveira, V., \& Franco, D. (2014). Museu de biodiversidade do cerrado: narração das memórias de um mediador. In: Atas ENEBIO 5, São Paulo.

De Queiróz, H., \& Fernandes, A. (2015). "Conversas de aprendizagem" em zoológicos e suas relações com a conservação da Biodiversidade. In: Atas do $X$ Encontro Nacional de Pesquisa em Educação em Ciências - X ENPEC, Águas de Lindóia.

Genzini, T., \& De Almeida, J. (2013). Comunicação pedagógica em museus de ciências. In: Atas do IX Encontro Nacional de Pesquisa em Educação em Ciências - IX ENPEC, Águas de Lindóia.

Kimble, J. (2013). Children learning about biodiversity at an environment Centre, a museum and at live animal shows. Studies in educational evaluation. 
Bio - grafía. Escritos sobre la Biología y su Enseñanza. ISSN 2027-1034

Edición Extraordinaria. p.p. 1078 - 1087

Memorias del IX Encuentro Nacional de Experiencias en Enseñanza de la Biología y la Educación Ambiental. IV Congreso Nacional de Investigación en Enseñanza de la Biología.

Kistler, F., \& Marandino, M. (2015). Identificando objetos em destaque em museus para análise de seu potencial educativo no ensino e a aprendizagem de ciências. In: Atas X Encontro Nacional de Pesquisa em Educação em Ciências - X ENPEC, Águas de Lindoia.

López, L. (2011). El aporte experiencial que ofrece una visita a la sala de ciencias naturales (SCN) del Museo Universitario de la Universidad de Antioquia (MUUA), en la apropiación de conceptos sobre la evolución biológica y la taxonomía animal desde una perspectiva ambiental: la contribución del aprendizaje informal y la mediación museistica. Trabajo de grado - Universidad de Antioquia, Antioquia.

Marandino, M., Dias, A., \& Mortensen, M. (2009). Discussing biodiversity in dioramas: a powerful tool to museum education. ICOM Natural History Committee Newsletter, 29, 30-36.

Marandino, M., \& Diaz, P. (2011). La biodiversidad en exposiciones inmersivas de museos de ciencias: implicaciones para educación en museos. Enseñanza de las Ciencias, 29(2), 221-236.

Moraes, R. (1999). Análise de Conteúdo. Revista Educação, 22(37), 7-31.

Moss, A., Jensen, E., \& Gusset, M. (2014). Evaluating the Contribution of Zoos and Aquariums to Aichi Biodiversity Target 1. Conservation biology, 0(0), 1-8.

Orozco, Y. (2016). La enseñanza y aprendizaje del concepto biodiversidad: Una mirada a través de la interpretación de propuestas teóricas y experiencias pedagógicas sobre este concepto. In: Memorias del III Congreso Internacional de Enseñanza de las Ciencias, Buenos Aires.

Topaz, M. (2016). Bioinspiration education at zoological institutions: an optimistic approach for innovation leading to biodiversity conservation. International Zoo Yearbook, 50, 112-124.

Wilson, E. (1988). Biodiversity. Washington: National Academy Press. 